\title{
MEASURING INSTRUMENTS USED AT THE RESEARCH AND ACCURACY OF DEFINING THE EFFICIENCY OF DRIVE SYSTEMS
}

\author{
Grzegorz Skorek \\ Gdynia Maritime University \\ Department of Engineering Sciences \\ Morska Street 81-87, 81-225 Gdynia, Poland \\ tel.: +48585586484 \\ e-mail: g.skorek@wm.umg.edu.pl
}

\begin{abstract}
The aim of the article is to look at the possibility of accurately determining the energy efficiency of drive systems thanks to the use of high quality sensors and measuring instruments. The types of measuring instruments used on the test stand are presented. The results of experimentally determined efficiencies and simulationally determined efficiencies of two hydrostatic systems with throttling control were compared, which are fed with a constant capacity pump. The choice of the analysed systems is not accidental. There is still a view in the literature about limited possibilities of energy systems with proportional control. The research stand was very carefully designed and made. The applied measuring instruments were characterized by high accuracy of measurements. The issues related to the determination of energy losses and energy efficiency of the engine or drive system, which should be determined as dependent on the physical quantities independent of these losses, were also discussed. For laboratory verification, measurement methods were developed, the test stand was adapted and automated. It consists of tested system and loaded system. The measurements during the tests were saved on the computer disk. In order to be able to compare the efficiency of the overall system with the efficiency obtained on the basis of the simulation, coefficients $k_{i}$ determining the energy losses of individual elements of the system were calculated. The research showed a large convergence of the mathematical description of energy losses in the elements of the system and the efficiency of the system with reality. The mathematical model enables accurate simulation determination of the energy efficiency of the system at each point of its field of operation, i.e. at each speed and load of the controlled hydraulic motor. The range of motor speed and load variation can also be accurately determined simulationally.
\end{abstract}

Keywords: energy efficiency, accuracy, measurements, measurement results, measuring instruments

\section{Introduction}

The article aims to present the measuring instruments used on the test stand enabling precise determination of the energy efficiency of drive systems, thanks to precise measurements. The measurements during the tests were recorded on the computer disk. In order to be able to compare the efficiency of the overall system with the efficiency obtained on the basis of the simulation, $\mathrm{k}_{\mathrm{i}}$ coefficients determining the energy losses of individual elements of the system were calculated.

The article is based on the works [1-3] aimed at describing specific issues related to determining the efficiency of the structure and also aims at presenting the accuracy with which the simulation model of the energy behaviour of the drive system and hydrostatic control maps reality.

The studies took into account the impact of energy losses in the pump, motor and conduits, decrease of the speed of the electric motor driving the pump, characteristics of the overflow valve, load and motor speed.

The analysis of the efficiency of individual elements of the structure examined and the comparison of the simulation efficiency determined with the laboratory obtained were possible thanks to the development by prof. Z. Paszota, the mathematical model of losses and energy efficiency of the considered system [1-3]. On the basis of a mathematical model, as well as models 
related to other structures, energy simulation programs for hydrostatic drive and control systems were created. In addition to obtaining an image of the efficiency of the systems, which is the effect of hydraulic motor operation parameters and operating conditions of the entire system, it is possible to compare and assess the impact of various structures.

The choice of the analysed system is not accidental. There is still a view in the literature about limited energy efficiency possibilities of a system with proportional control.

\section{Constant-pressure and variable pressure system with throttling control}

The most common in practice is system with throttling control of a linear hydraulic motor (Fig. 1), in which the proportional directional control valve is supplied with a constant capacity pump cooperating with an overflow valve stabilizing the constant supply pressure $p=$ cte equal to the nominal pressure. This system achieves high-energy efficiency, similar to the efficiency of the volume control system with variable capacity pump, at the point with maximum values of the speed coefficient and the motor load coefficient [4]. With the decreasing load $F_{M}$ of the motor, and especially with the deceleration of its speed $v_{M}$, the efficiency $\eta$ of the constant pressure system with the series throttling control sharply decreases (Fig. 8) [5].

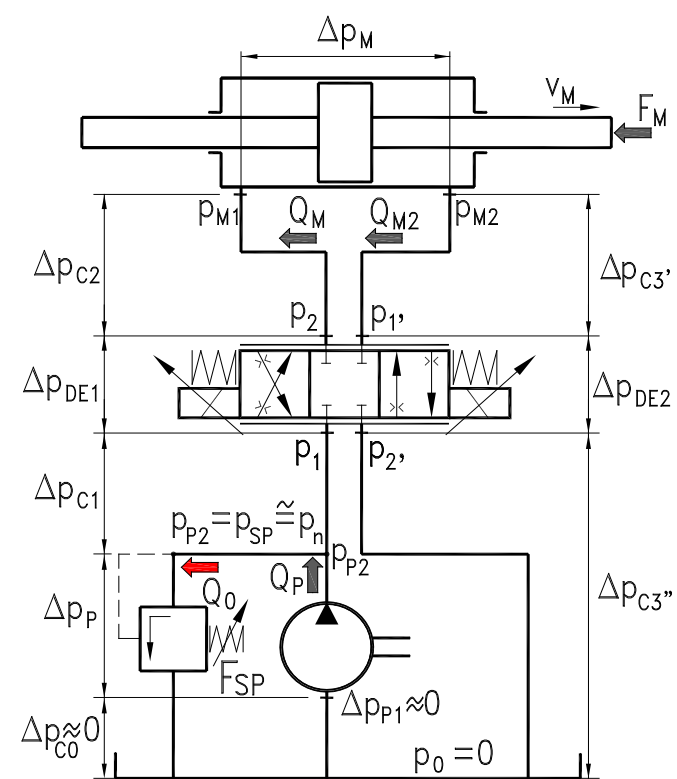

Fig. 1. Diagram of the tested system at constant pressure $-p=$ cte structure

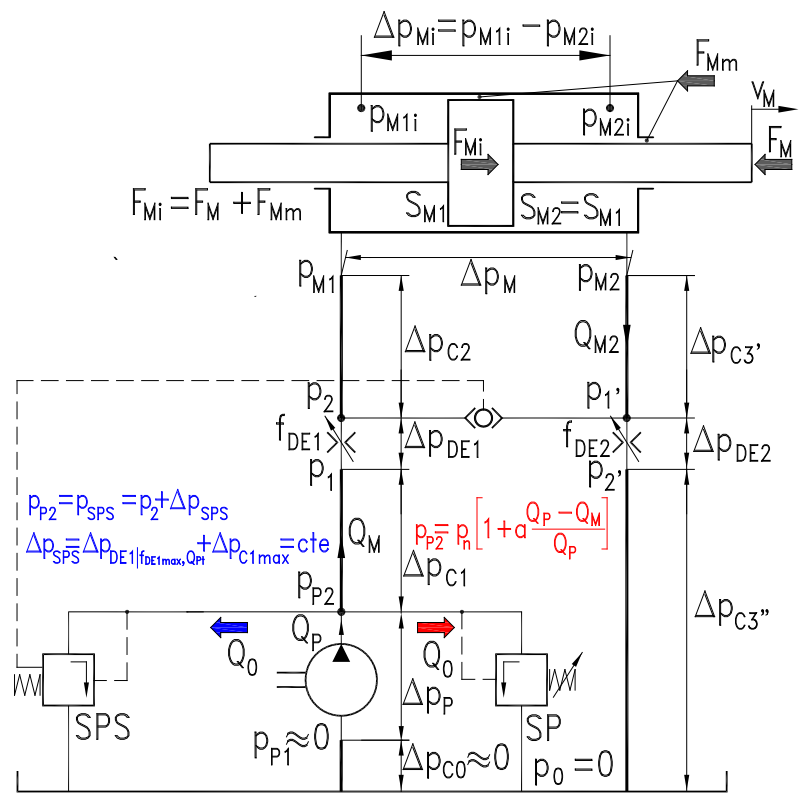

Fig. 2. Diagram of the tested system with proportional valve fed with constant pump cooperating with a controlled overflow valve in a variable pressure system $-p=v a r$

There are possibilities to reduce energy losses in elements of a system with proportional control (in the pump, in a throttling control unit and in a hydraulic motor, especially in a linear motor), and thus the possibility of increasing the energy efficiency of the system with the throttling control valve.

The hydraulic system of the drive and proportional control of the linear hydraulic motor, for example, can be supplied with a constant capacity pump cooperating with an overflow valve controlled by a pressure $\mathrm{p}_{2}$ at the outlet from the control valve to the cylinder. The variable pressure system $p=\operatorname{var}$ (Fig. 2) enables reduction of losses in the pump, in the control unit and in the hydraulic linear motor [5].

In the variable pressure system $p=$ var, the structural pressure and volume losses in the throttling control unit can be severely reduced, the mechanical losses in the cylinder and the pump as well as volumetric losses in the pump. The mathematical description of losses and efficiency was presented in $[1,4,5]$. 


\section{Measuring instruments}

The measurement of the force $\mathrm{F}_{\mathrm{M}}$ of mechanical losses - friction forces in the tested cylinder, operating at a constant speed, was made using the indirect method, as there is no device enabling the measurement to be carried out using the direct method. This made it necessary to use high quality transducers for measuring force and pressure.

The LabVIEW 6.0 program was used to read and record the measurement results. The element connecting the computer with transducers was measurement card type PCI 1713 from Advantech. During the tests, four analogue pressure signals, force $\mathrm{F}_{\mathrm{M}}$ signal on the piston rod and a position signal were measured, on the basis of which the speed $\mathrm{v}_{\mathrm{M}}$ of the piston rod was determined.

During continuous operation of the system, in which the cylinder performs reciprocating movements, the speed of the cylinder changes. Speed jumps are caused by piston rod micro movements. This dispersion comes from the measurement method and depends on how the displacement is measured and at what time intervals it is implemented.

Due to the fact that the cylinder moves first, accelerates first, then moves at a constant speed, then brakes to finally stand, the average value is taken as the result of the measurement in which the piston rods and the cylinder piston move at a constant speed.

\subsection{Pressure transmitters}

On test stand were used pressure transmitters of type MPS-03 and class 0.1 with a measuring range up to $24 \mathrm{MPa}, 6.3 \mathrm{MPa}$ and $1.6 \mathrm{MPa}$ (Fig. 3). Each transmitter is equipped with a display and a potentiometer, which are useful at calibrating the initial settings. The measurement results were sent to the computer and recorded up to date.

Every transmitter type MPS-03 with a measuring range up to $24 \mathrm{MPa}$ have a small measurement error, which is $0.07 \%$ and has a linear characteristic. For better accuracy of measurements and for the measurement of lower pressures, in the range below $6 \mathrm{MPa}$, transducers with a smaller measuring range were used - up to $6.3 \mathrm{MPa}$.

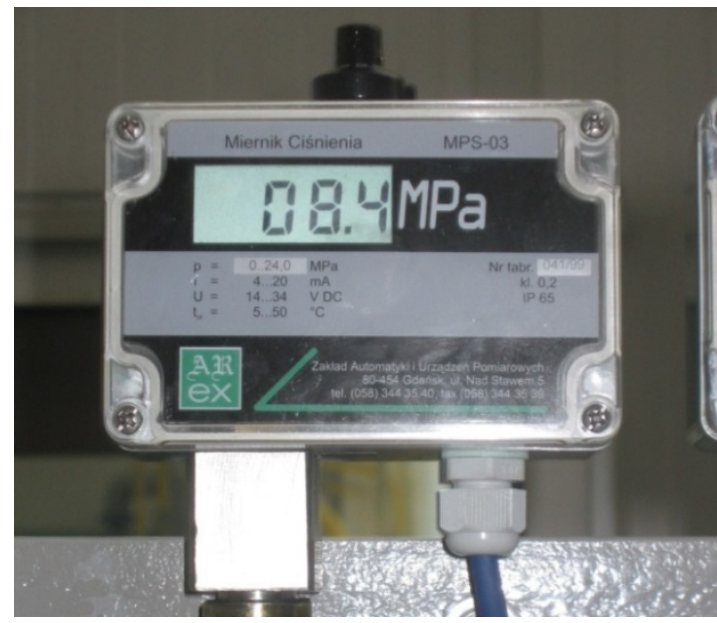

Fig. 3. Pressure transmitter

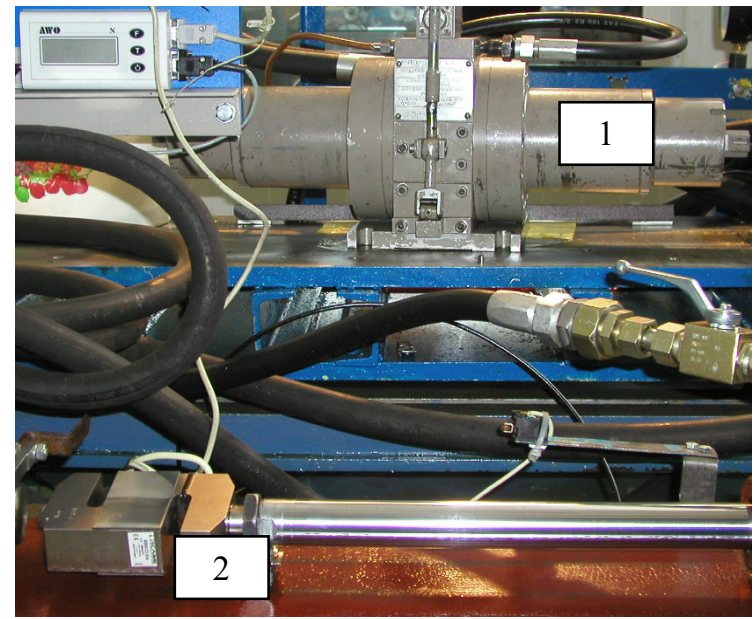

Fig. 4. Piston flowmeter 1, force transducer 2

\subsection{Flowmeter}

A flowmeter type PT200 and class 0.2 has been used to measure the flow rate in the system components $(1-$ Fig. 4$)$. The maximum measuring error of the device measuring the flow rate up to $0.0033 \mathrm{~m}^{3} / \mathrm{s}$ (up to $200 \mathrm{dm}^{3} / \mathrm{min}$ ) is $\pm 0.0000066 \mathrm{~m}^{3} / \mathrm{s}\left( \pm 0.4 \mathrm{dm}^{3} / \mathrm{min}\right.$ ).

This flowmeter together with the receiver type $\mathrm{CMH}-2 \mathrm{C}$, made at the Gdansk University of Technology, enables accurate measurements of oil flow in high-pressure hydraulic systems on test 
stand in which mineral oil is used as a working medium. The parameters like viscosity, pressure, and temperature of liquids have practically no influence on flowmeter type PT200.

This flowmeter consists of a mechanical and electronic assembly. A piston is placed in the measuring cylinder. Coaxial to the cylinder is placed the measuring plunger, in which three reed sensors have been installed. The contacts of the reed contacts close in succession when they are in the magnetic field of the magnet placed in the moving plunger.

The principle of measuring the volumetric flow rate of a liquid with a piston flow meter is based on measuring time $t$, in which the piston measures the reference volume $V$. The measurement of these parameters and the calculation of the capacity $\mathrm{Q}$ value is made by the receiver and meter type $\mathrm{CMH}-2 \mathrm{C}$.

\subsection{Linear displacement transducer}

Linear displacement transducer $(1$ - Fig. 5), connected to the piston rod of the tested system, makes it possible to determine the speed $\mathrm{V}_{\mathrm{M}}$ of the piston rod and, taking into account the active surface of its piston, determine the intensity of flow $\mathrm{Q}_{\mathrm{M}}$ of the liquid stream flowing into it.

On the test stand was used a transformer linear displacement transducer series PLx 500 by Peltron, which is designed for measurements of large linear displacements. The basic unit of the device is a differential transformer located in a cylindrical housing. In the transformer coil, the magnetic core moves from the position of which the output signal depends. The transducer's design allows the core to be inserted from any of its sides. The maximum non-linearity of the device is $\pm 0.01 \%$. Linear displacement transducer was connected to signal amplifier and multimeter.

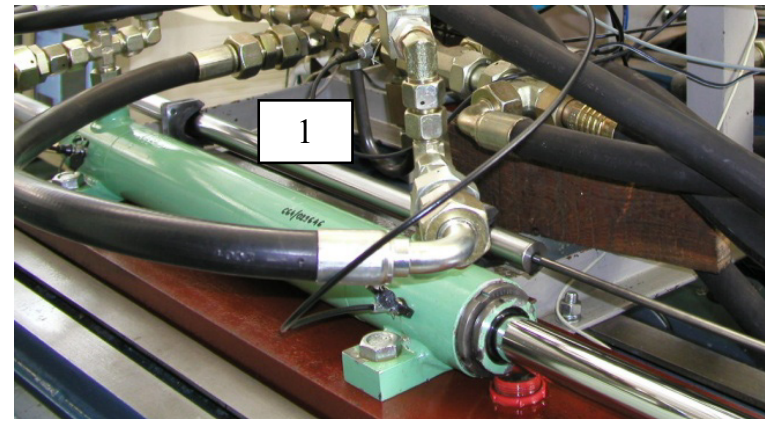

Fig. 5. Linear displacement transducer 1

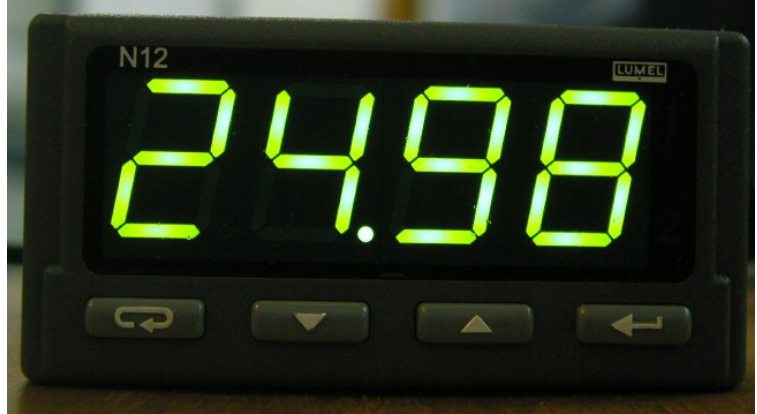

Fig. 6. View of the speed sensor meter

\subsection{Force transducer}

The extensometer force transducer ( 2 - Fig. 4$)$ of type FT5109L was used on test stand to measure the force $F_{M}$ loading the tested cylinder. It was cooperated with the amplifier AT-5230 from AWO, having the accuracy class of 0.1. Due to the fact that the transducer is designed to measure compressive forces, it was installed between the test and loading cylinders. The transducer works on the principle of measuring, by means of strain gauge, elastic deformation of the measuring element under the influence of the applied force. Changes in ambient temperature are compensated in the sensor's electronical system. The deformation of the measuring element causes a change in the resistance in the extensometer system, transformed in the electronic system of the amplifier into an output signal proportional to the applied force. The transducer, connected to the amplifier, is a force-measuring device which after calibration prior to each measurement measures and transmits to the computer results in the range from $1 \mathrm{~N}$ to $40 \mathrm{kN}$ with an accuracy of $\pm 1 \mathrm{~N}$.

\subsection{Speedometer}

For measuring the rotational speed $n_{P}$ of the pump shaft, the transducer was used together with the programmable meter N12B from LUMEL (Fig. 6). The instrument error is $\pm 0.1 \%$ of the 
displayed value. The N12B type digital meter is designed for exposure of the measurement result sent via the RS 485 interface.

\subsection{Torque transmitter}

In order to record the torque $\mathrm{M}_{\mathrm{P}}$ on the pump shaft, a torque transmitter was installed of type AB12 from Hottinger Baldwin Messtechnik (Fig. 7a and b). It can measure the torque value in the range from 0 to $500 \mathrm{Nm}$. The device has a class of 0.1 , so it works with an accuracy of $\pm 0.5 \mathrm{Nm}$.

a)

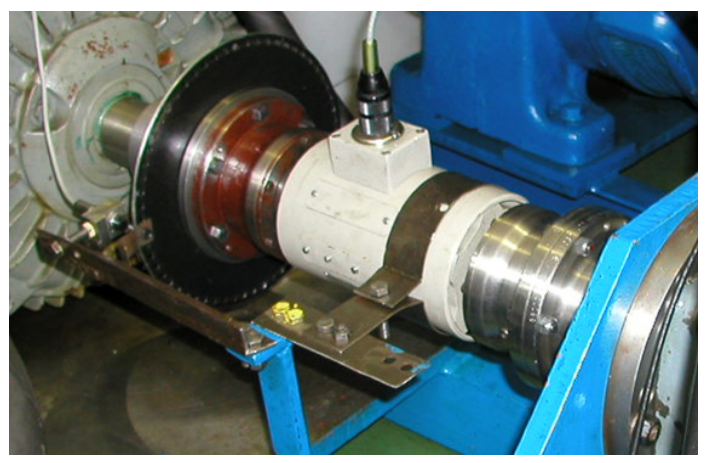

b)

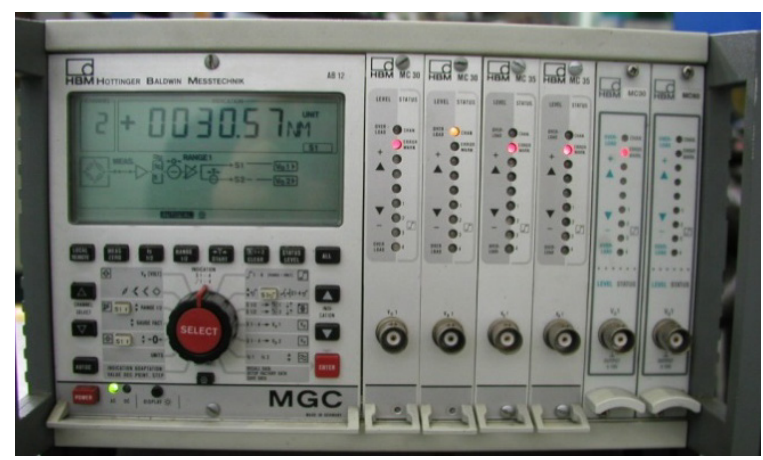

Fig. 7. A torque transducer consisted of device with extensometers installed on shaft (a) and device for reading recorded parameters (b)

\section{Energy efficiency of hydrostatic systems determined by simulation based on laboratory investigated coefficients $k_{i}$ of losses}

Figure 8 shows the overall efficiency $\eta$ of the constant-pressure system $p=$ cte (Fig. 1) and variable pressure system $p=\operatorname{var}$ (Fig. 2) determined by simulation. Fig. 8 shows also the thin dashed lines of the overall efficiency $\eta$ of systems for the maximum use of the pump efficiency system, i.e. in a situation in which the $\mathrm{Q}_{\mathrm{M}}$ intensity of the stream directed to the cylinder through the proportional directional control valve approaches the pump Qp capacity. In this case, it is possible to achieve the maximum energy efficiency $\eta$ of both systems equal to $\eta=0.746$ at $\overline{\mathrm{M}}_{\mathrm{M}}=0.855$ $\left(\mathrm{F}_{\mathrm{M}}=25650 \mathrm{~N}\right)$ and $\bar{\omega}_{\mathrm{M}}=0.939\left(\mathrm{v}_{\mathrm{M}}=0.380 \mathrm{~m} / \mathrm{s}\right)$.

By the cylinder load $\overline{\mathrm{M}}_{\mathrm{M}}$ coefficient equal to $\overline{\mathrm{M}}_{\mathrm{M}}=0.863\left(\mathrm{~F}_{\mathrm{M}}=25890 \mathrm{~N}\right)$, the efficiency $\eta$ of both systems, for speed coefficient $\bar{\omega}_{\mathrm{M}}$ equal to $\bar{\omega}_{\mathrm{M}}=0.063(\mathrm{v} \mathrm{M}=0.025 \mathrm{~m} / \mathrm{s})$ is only about $\eta \approx 0.047$. In turn, the efficiency $\eta$ of both systems, with the same load coefficient $\overline{\mathrm{M}}_{\mathrm{M}}$ equal to $\overline{\mathrm{M}}_{\mathrm{M}}=0.863\left(\mathrm{~F}_{\mathrm{M}}=25890 \mathrm{~N}\right)$ and at a common speed $\bar{\omega}_{\mathrm{M}}$ coefficient equal to $\bar{\omega}_{\mathrm{M}}=0.875$ $\left(\mathrm{v}_{\mathrm{M}}=0.350 \mathrm{~m} / \mathrm{s}\right)$ of speed, reaches the approximation value of $\eta \approx 0.692$ [5].

From the point of view of the overall efficiency $\eta$ of the system, the greatest gain is at value of the cylinder load coefficient $\overline{\mathrm{M}}_{\mathrm{M}}$ of approximately $\overline{\mathrm{M}}_{\mathrm{M}} \approx 0.200\left(\mathrm{~F}_{\mathrm{M}} \approx 6000 \mathrm{~N}\right)$, with speed coefficient $\bar{\omega}_{M}$ equal to $\bar{\omega}_{M}=0.875(\mathrm{vM}=0.350 \mathrm{~m} / \mathrm{s})$. The overall efficiency $\eta$ of the $\mathrm{p}=$ cte system is then $\eta=0.158$, and the overall efficiency of the system $p=\operatorname{var}-\eta=0.413$, which is around 2.6 times higher than the efficiency of the constant pressure system. In this zone, the medium load zone begins.

\section{Accuracy of determining the efficiency of systems by simulation method}

In order to verify the mathematical models proposed in the simulation method for determining the energy efficiency of the motor proportional control system and to evaluate the accuracy of this method, it was necessary to compare the energy efficiency $\eta$ results of the constant pressure system $p=$ cte and variable pressure system $p=$ var determined simultaneously with the results of 
direct accurate laboratory tests (Fig. 8). Therefore, these results were compared at selected values of the speed coefficient $\bar{\omega}_{M}$ and the load coefficient $\bar{M}_{M}$ of the cylinder.

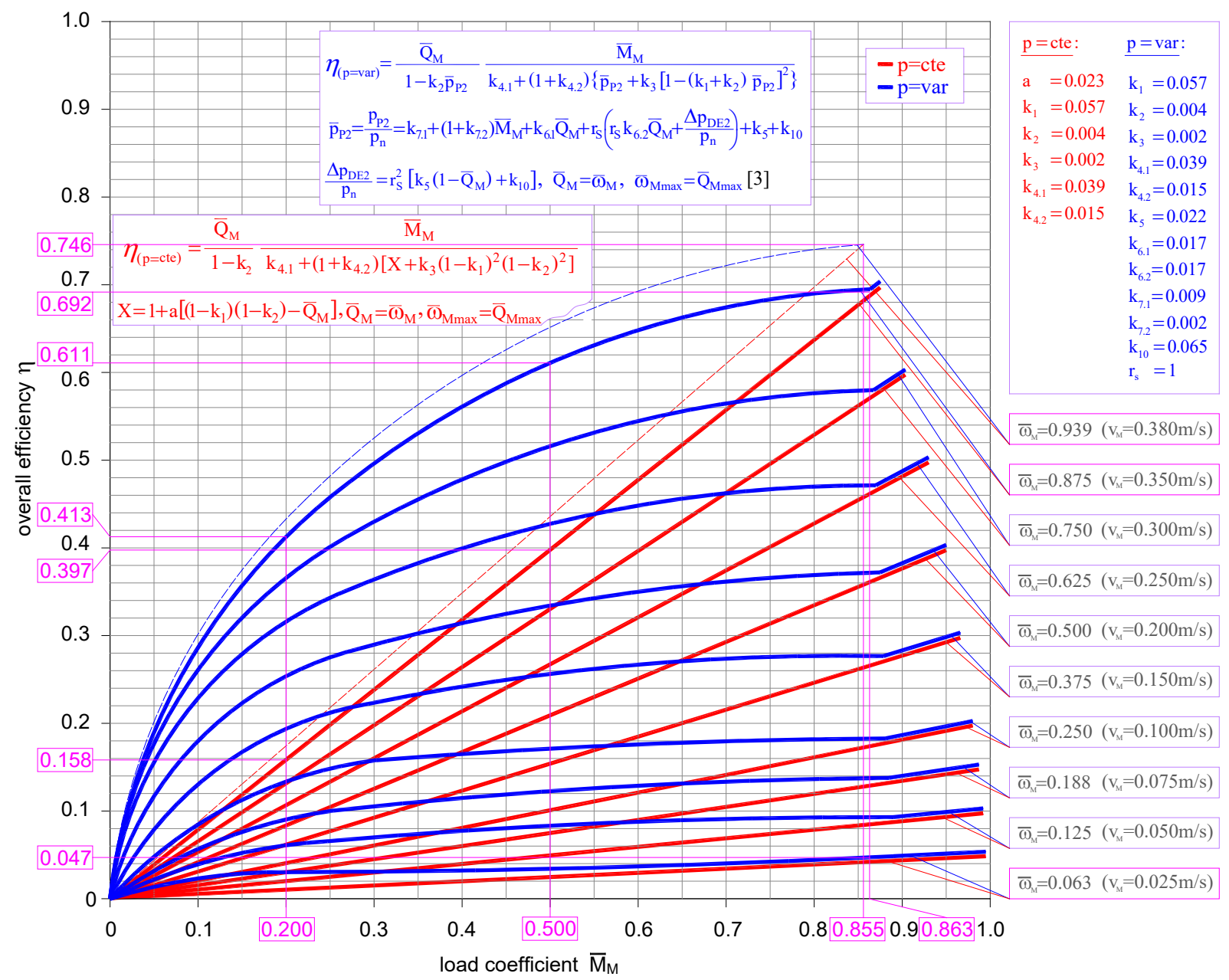

Fig. 8. The dependence of the overall efficiency $\eta$ of the constant pressure system $(p=c t e)$ and the variable pressure system $\left(p=\right.$ var) on the load coefficient $\bar{M}_{M}$ at different speed coefficients $\bar{\omega}_{M}$ of the cylinder; overall efficiency $\eta$ of the systems defined by simulation based on the laboratory investigated coefficients $k_{i}$ of losses; viscosity $v_{n}=35 \mathrm{~mm}^{2} / \mathrm{s}$

The absolute error of the simulation model of the mathematical overall energy efficiency of hydrostatic drive with proportional control of the hydraulic motor (hydraulic cylinder) supplied by a constant-capacity pump in the constant pressure system $p=$ cte (as the difference between experimental and simulation results) is of the order from -0.0036 to +0.0009 , which is in the order of $0.4 \%$. The absolute error of determining the overall efficiency of the system working in the variable pressure system $p=$ var as the difference between experimental and simulation results ranges from -0.0014 to +0.0108 , which is in the order of $1 \%$.

The results of laboratory verification tests confirm with high accuracy the theoretical and mathematical descriptions of simulations of energy losses in elements of hydraulic servo systems or systems with a proportional directional control valve operating in the supply system $p=$ cte and $\mathrm{p}=$ var.

\section{Conclusions}

1) The obtained test results, thanks to the use of accurate measuring instruments, allow drawing conclusions that the simulation method for determining the overall energy efficiency of 
a hydrostatic drive system with proportional control of a hydraulic motor supplied with a constant capacity pump in a constant pressure system $\mathrm{p}=$ cte and variable pressure system $\mathrm{p}=$ var represents reality with very high accuracy.

2) The tested structures $(p=$ cte and $p=$ var) of hydrostatic drives with throttling control of linear speed, fed with a constant capacity pump, can achieve, with maximum load $F_{M \max }\left(\overline{\mathrm{M}}_{\mathrm{M} \max }=\right.$ $=0.863)$ and simultaneous maximum speed $\mathrm{V}_{\mathrm{Mmax}}\left(\bar{\omega}_{\mathrm{M} \max }=0.875\right)$ of this hydraulic motor, the same maximum overall efficiency $\eta_{\max }$ equal $\eta_{\max }=0.692$ of the system.

3) The energy efficiency tests with speed series throttling control of the hydraulic motor (systems with throttling control valve) confirm that their energy efficiency can be much higher than the values given again and again in the literature of the subject.

4) The results of laboratory verification tests (direct laboratory tests of energy efficiency of systems) confirm the high accuracy of mathematical descriptions of simulations of energy losses in elements of systems with proportional control valve working in the supply system $\mathrm{p}=$ cte and $\mathrm{p}=$ var.

5) Simulation tests of motors and hydrostatic drive systems replace research in the laboratory. It is possible to apply these formulas in a different case, having verified with practice, with other loss coefficients $\mathrm{k}_{\mathrm{i}}$ characterizing various structural solutions of the system elements. Mathematical formulas that describe energy efficiency evaluate the reality with great accuracy.

6) In the simulation method of determining the energy efficiency of hydrostatic drive systems, the characteristics of pumps and hydraulic motors are limited only to determining the coefficients $\mathrm{k}_{\mathrm{i}}$ of energy losses in these elements.

\section{References}

[1] Paszota, Z., Model of losses and energy efficiency of the hydraulic system with proportional control of the hydraulic cylinder supplied with a constant capacity pump in a variable pressure system, Chapter in monograph: „Research, construction, manufacture and operation of hydraulic systems”, pp. 145-162, Komag, Gliwice 2005.

[2] Paszota, Z., On power stream in motor or drive system, Polish Maritime Research, Vol. 4, pp. 93-98, Gdansk 2016.

[3] Paszota, Z., Energy losses in hydrostatic drive. Drive investigation method compatible with diagram of power increase opposite to the direction of power flow, LAP Lambert Academic Publishing, Saarbrucken 2016.

[4] Skorek, G., Energy characteristics of the hydraulic system with proportional control of cylinder, fed by a constant capacity pump in a constant pressure and variable pressure system (in Polish), Doctor dissertation, Gdansk University of Technology, Faculty of Ocean Engineering and Ship Technology, Gdansk 2010.

[5] Skorek, G., Energy efficiency of a hydrostatic drive with proportional control compared with volumetric control, Polish Maritime Research, Vol. 3, pp. 14-19, Gdansk 2013.

Manuscript received 06 March 2019; approved for printing 21 June 2019 